\title{
Stone-Wales Defect and Vacancy-Assisted Enhanced Atomic Orbital Interactions Between Graphene and Ambient Gases: A First- Principles Insight
}

\author{
Jeevesh Kumar, Ansh, and Mayank Shrivastava*
}

Cite This: ACS Omega 2020, 5, 31281-31288

Read Online

ACCESS | Lلll Metrics \& More | 国 Article Recommendations | st Supporting Information

ABSTRACT: Graphene has magnificent fundamental properties for its application in various fields. However, these fundamental properties have been observed to get perturbed by various agents like intrinsic defects and ambient gases. Degradation as well as p-type behavior of graphene under an ambient atmosphere are some of the properties that have not yet been explored extensively. In this work, interactions of different ambient gases, like $\mathrm{N}_{2}, \mathrm{O}_{2}, \mathrm{Ar}, \mathrm{CO}_{2}$, and $\mathrm{H}_{2} \mathrm{O}$, with pristine and defective graphene are studied using density functional theory (DFT) computations. It is observed that while the pristine graphene is chemically and physically inert with ambient gases, except for oxygen, its interaction with these ambient gases increases significantly in the presence of carbon vacancies and

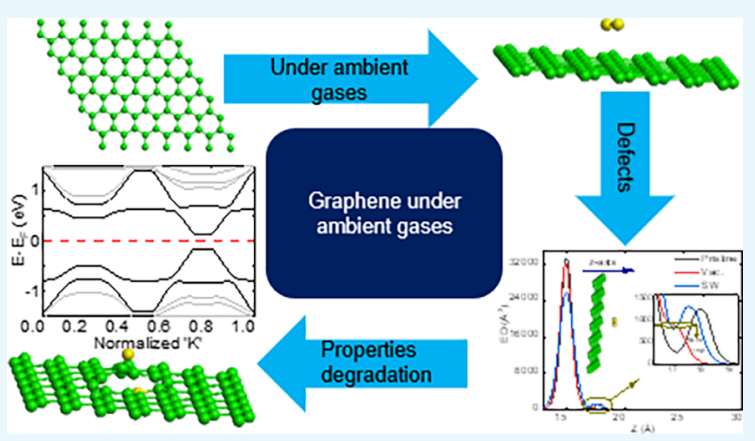
Stone-Wales (SW) defects. We report that $\mathrm{Ar}$ and $\mathrm{N}_{2}$ are apparently not inert with defective graphene, as they also influence its fundamental properties like band structure, mid gap (trap) states, and Fermi energy level. We have also found that while oxygen makes pristine graphene p-type, the phenomenon amplifies in the presence of SW defects. Besides, in the presence of carbon vacancies, $\mathrm{N}_{2}, \mathrm{H}_{2} \mathrm{O}$, and $\mathrm{CO}_{2}$ also make the graphene monolayer p-type. Among ambient gases, oxygen is the real performance and reliability killer for graphene. Its reaction is seeded by a carbon vacancy, which initiates its degradation by local formation of graphene oxide.

\section{INTRODUCTION}

Graphene, since its discovery, ${ }^{1}$ has attracted remarkable attention from industry and the research community due to its excellent electrical, ${ }^{2}$ thermal, ${ }^{3}$ mechanical, ${ }^{4}$ optical, ${ }^{5}$ and magnetic ${ }^{6}$ properties. However, these properties of graphene are strongly influenced by some of the major atmospheric gases. ${ }^{7-12}$ Ryu et al. ${ }^{7}$ as well as Liu et al. ${ }^{8}$ and Shim et al. ${ }^{9}$ have investigated p-type doping of graphene by oxygen and water, respectively. The authors, however, have not given detailed theoretical insights into the reported interactions. Yang et al. ${ }^{10}$ have studied the influence of atmospheric gases on electrical transport in graphene; however, the authors did not discuss the fundamental cause of the reported changes in doping and mobility. Graphene over a metal substrate shows complex interaction mechanisms with gases, like intercalation and splitting of water on a $\mathrm{Cu}(111)$ substrate, ${ }^{13}$ decomposition of water on a $\mathrm{Ni}(111)$ substrate, ${ }^{14}$ nanobubble implantation of argon on a $\mathrm{Ni}(111)$ substrate, ${ }^{15}$ and sequestration of carbon monoxide on a $\mathrm{Ru}(0001)$ substrate. ${ }^{16}$ Similar behavior of graphene, however, cannot be expected after its transfer due to the absence of catalysis action of corresponding metals. Detailed investigations to probe the impact of ambient gases on graphene are not available yet, except for preliminary studies like the study by Grosjean et al., ${ }^{17}$ on the effect of hydroxide, ${ }^{17}$ its interaction with water in the study by Freitas et al. ${ }^{18}$ as well as Al-Hamdani et al., ${ }^{19}$ and adsorption of $\mathrm{CO}_{2}$ in the study by Lee et al. ${ }^{20}$ These studies, ${ }^{17-20}$ however, also did not explore the impact of the studied gases on the fundamental properties of graphene like band structure, Fermi energy, and mid gap (trap) states, which would directly influence the electrical and optoelectronic properties of graphene-based devices. Hence, theoretical insights into such environmental effects, p-type behavior of graphene, and its degradation in an ambient environment still remain to be probed in depth.

It is known that there are various structural defects that are inherently present in graphene after growth or device processing. ${ }^{21-23}$ These defects, especially void and StoneWales (SW), deteriorate electrical, ${ }^{24,25}$ mechanical, ${ }^{26,27}$ and thermal $^{28}$ properties of graphene. Defects in graphene also influence its interactions with various sensitive gases. ${ }^{29-35}$ Due to such intrinsic defects, abundant atmospheric gases, especially inert gases like $\mathrm{Ar}$ and $\mathrm{N}_{2}$, can also interact with graphene and influence its fundamental properties, as discussed

Received: September 27, 2020

Accepted: November 4, 2020

Published: November 25, 2020 

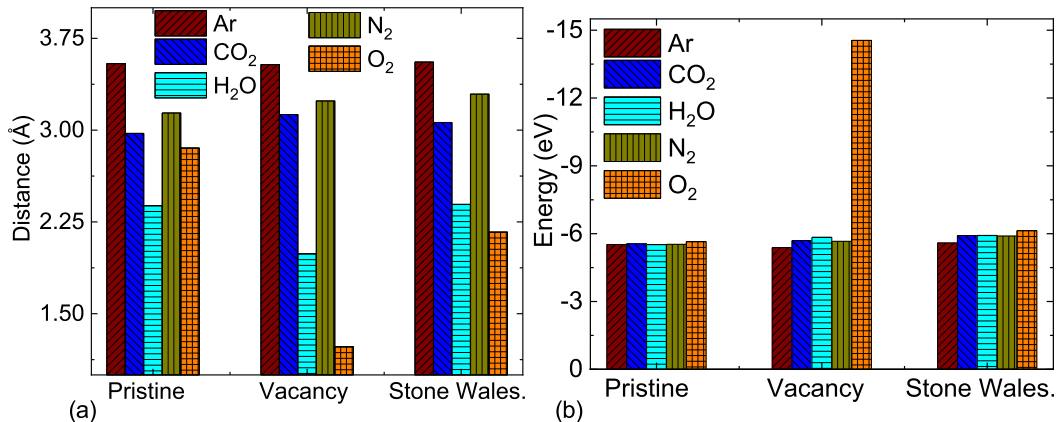

Figure 1. (a) Observed distance of various molecules from graphene surfaces, poststructure optimization for minimum energy. (b) Energy of various graphene-molecule systems explored, extracted poststructure optimization for minimum energy.
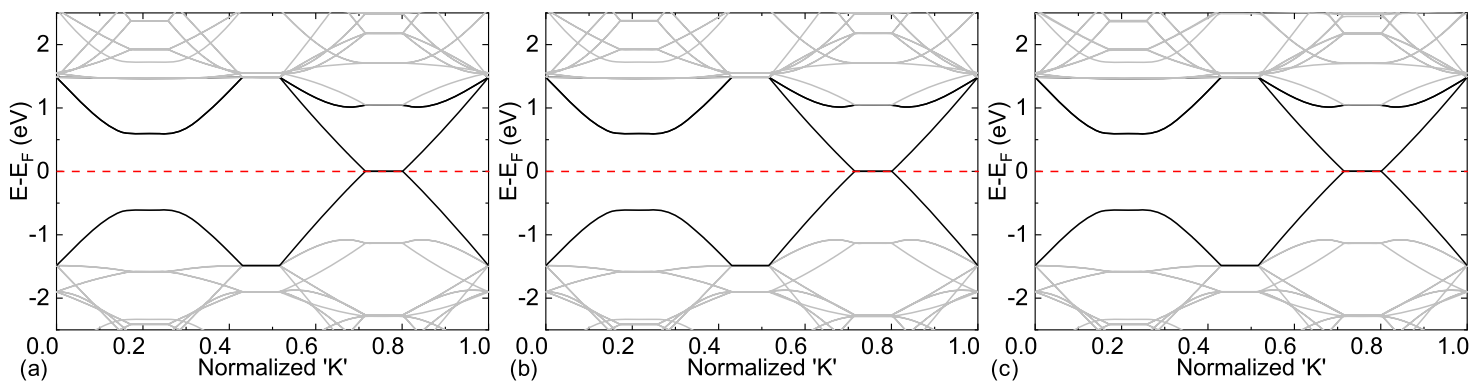

Figure 2. Band structures of a $7 \times 7$ supercell of (a) pristine graphene, (b) a pristine graphene-Ar system, and (c) a pristine graphene- $\mathrm{N}_{2}$ system.

later in this work. The impact of defects on interactions of ambient gases with graphene has not been investigated yet, except for investigations done by $\mathrm{Li}$ et al., ${ }^{30}$ in which the authors probed defect-induced oxygen adsorption on graphene.

In this work, using density functional theory (DFT) computations, we have investigated the impact of ambient gases on the fundamental properties of graphene in the presence of a carbon vacancy and SW defects. Nitrogen $\left(\mathrm{N}_{2}\right)$, oxygen $\left(\mathrm{O}_{2}\right)$, water $\left(\mathrm{H}_{2} \mathrm{O}\right)$, argon $(\mathrm{Ar})$, and carbon dioxide $\left(\mathrm{CO}_{2}\right)$ molecules have been selected for the study due to their abundant presence in the atmosphere. We have explored how chemical and physical interactions of these gases with graphene are influenced by a carbon vacancy and SW defects. We have also investigated the strength of inertness of $\mathrm{Ar}$ and $\mathrm{N}_{2}$ toward graphene due to these defects. Furthermore, we have studied atomic orbital interactions between the gases and carbon atoms at the defect sites. Moreover, influences of the fundamental properties of graphene like band structure, Fermi energy, and mid gap (trap) states are explored too. Using these explorations, we have explained why graphene behaves as a p-type material under ambient conditions. Finally, we have also revealed that oxygen in the presence of defects makes graphene thermodynamically less stable by initiating graphene degradation through graphene oxide formation at the carbon vacancy site.

\section{RESULTS AND DISCUSSION}

Bonding Features of the Interactions. Carbon vacancies and Stone-Wales defects are prominent point defects in CVD-grown graphene. ${ }^{22}$ It is worth exploring how defects would affect interactions of ambient gases with graphene, which can potentially perturb its fundamental properties. To explore this, ambient gaseous molecules were optimized over different graphene supercells (Supporting Information (SI), Figure S3) to achieve their minimum energy conditions. It is observed that all of the molecules sit over pristine graphene within the van der Waals (vdW) attraction regime. Distances of $\mathrm{Ar}, \mathrm{CO}_{2}, \mathrm{H}_{2} \mathrm{O}, \mathrm{N}_{2}$, and $\mathrm{O}_{2}$ from the pristine graphene surface are $\sim 3.54, \sim 2.97, \sim 2.38, \sim 3.14$, and $\sim 2.85 \AA$, respectively, as shown in Figure 1a. Binding energies of all of the molecules with different graphene surfaces (Figure 1b) were calculated using eq 1 .

$$
\Delta E=E_{\text {sys }}-\left(E_{\text {molecule }}+E_{\text {graphene }}\right)
$$

where $E_{\text {sys }}$ is the energy of the optimized graphenemolecule system, while $E_{\text {molecule }}$ and $E_{\text {graphene }}$ are optimized energies of the corresponding molecule and graphene, respectively, under isolated conditions.Negative values of the binding energies represent exothermic interactions, which confirms that all of the interactions are thermodynamically favorable. In the case of pristine graphene, all of the molecules are bound with $\sim 5 \mathrm{eV}$ of energy, which signifies that their interaction is physical (vdW interaction) in nature.

On the other hand, when a vacancy is created in the pristine graphene (vac. graphene), it leaves behind an unbound orbital. We have found that the unbound orbitals can enhance interactions of graphene with different gaseous molecules. Ar, $\mathrm{CO}_{2}$, and $\mathrm{N}_{2}$ were found to have similar vdW interactions to vac. graphene as they were found to place themselves $\sim 3.53$, $\sim 3.12$, and $\sim 3.23 \AA$ above the graphene surface, respectively, with $\sim 5 \mathrm{eV}$ vdW energy. $\mathrm{H}_{2} \mathrm{O}$ has a relatively stronger vdW interaction with a bond distance of $\sim 1.99 \AA$ due to hydrogen bonding. Oxygen, however, shows a very different behavior than other gas molecules in the presence of vac. graphene. Oxygen releases an energy of $\sim 14.54 \mathrm{eV}$ per atom, during the interaction with the vacancy site, which is greater than combustion of one carbon atom $(\sim 10.44 \mathrm{eV})$, and therefore it comes under the covalent regime of the carbon-oxygen bond. 

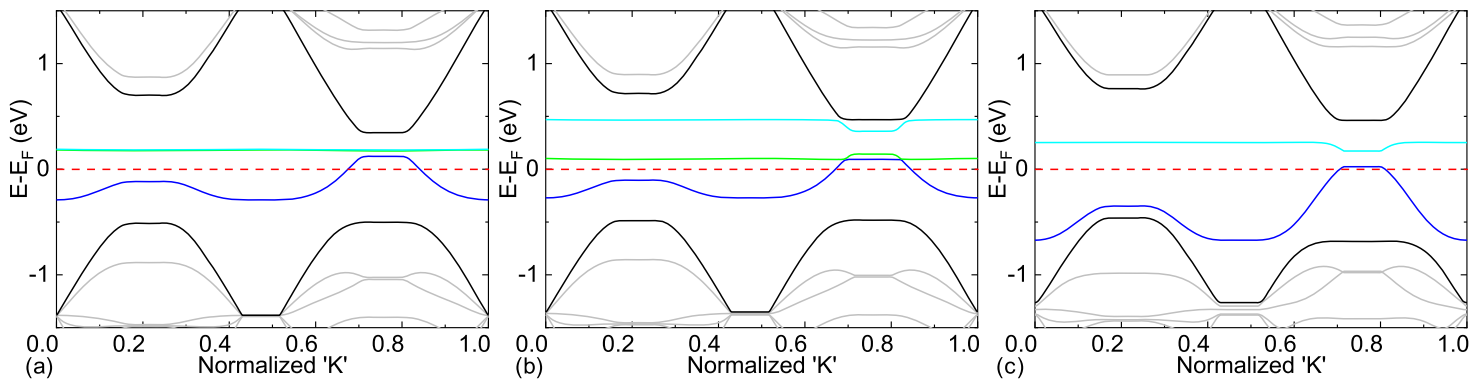

Figure 3. Band structures of a $7 \times 7$ supercell of (a) graphene with a $1 \mathrm{C}$ vacancy (vac. graphene), (b) a vac. graphene-Ar system, and (c) a vac. graphene- $\mathrm{N}_{2}$ system.
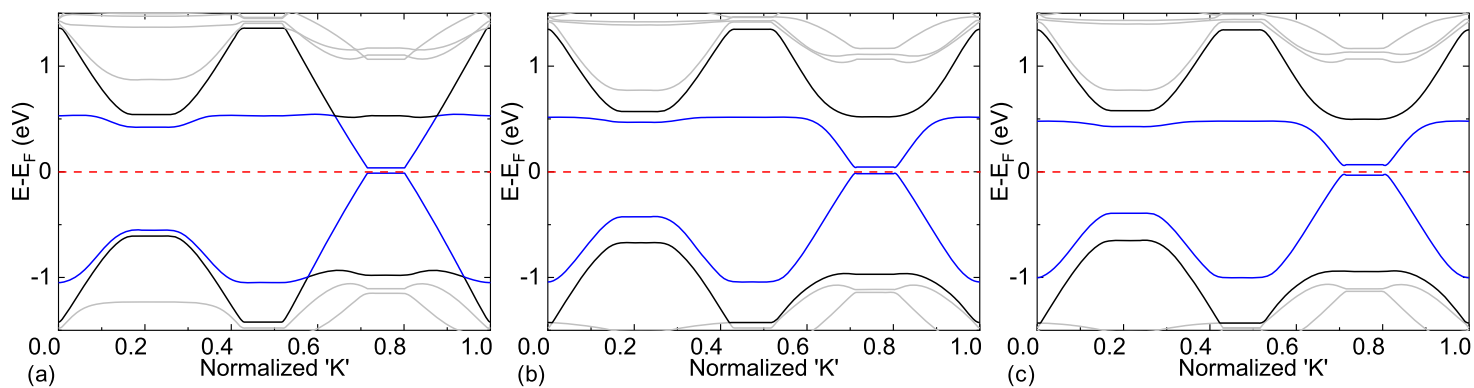

Figure 4. Band structures of a $7 \times 7$ supercell of (a) graphene with an SW defect (SW graphene), (b) an SW graphene-Ar system, and (c) an SW graphene- $\mathrm{N}_{2}$ system.
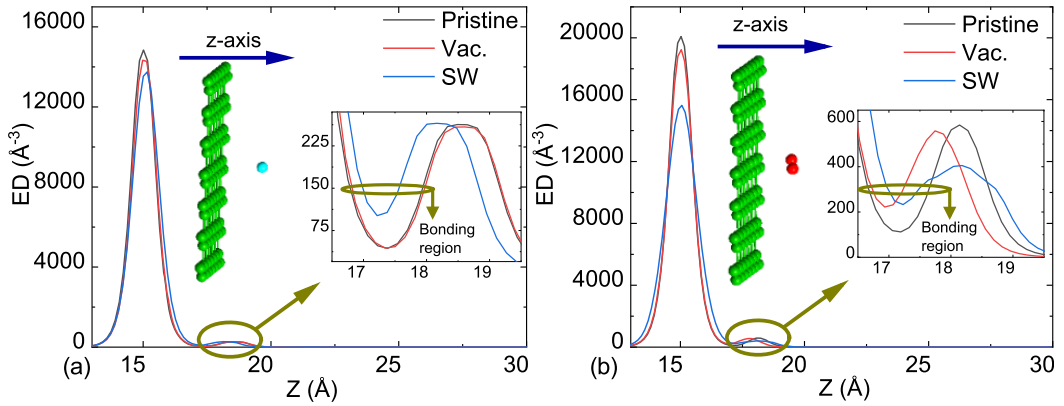

Figure 5. Electron density (ED) comparison of (a) graphene-Ar and (b) graphene- $\mathrm{N}_{2}$ systems for pristine and defective graphene cases. Insets show respective zoomed-in plots near the overlap regions. The $z$-axis is along the plane normal to graphene's plane and directed toward an $\mathrm{Ar}$ or $\mathrm{N}_{2}$ atom.

In the case of graphene with an SW defect, all of the molecules were found to be placed above the graphene surface having a vdW force of attraction between them (by releasing energy $\sim 5.5 \mathrm{eV}$ ). Distances of $\mathrm{Ar}, \mathrm{CO}_{2}, \mathrm{H}_{2} \mathrm{O}, \mathrm{N}_{2}$, and $\mathrm{O}_{2}$ from the graphene surface are $\sim 3.55, \sim 3.06, \sim 2.39, \sim 3.29$, and $\sim 2.16 \AA$, respectively (Figure $1 \mathrm{~b}$ ). When the gas molecules approach the surface at the SW site, the graphene plane becomes curved near the interaction site to maintain a vdW distance with the exposed molecule (SI, Figure S3).

Are $\mathrm{N}_{2}$ and Ar Gases Really Inert? Nitrogen and argon gases are extensively used to provide an inert environment during nanomaterial processing and characterizations. Although we have discussed in the previous section that these gases are not under a chemical bonding regime with graphene, however, we need to explore whether the gases are inert with graphene or they can perturb some of the fundamental properties of graphene, like band structures, Fermi levels, and position of energy states due to long-range interactions. We also need to understand influences of the point defects on such long-range interactions.
Nitrogen and argon were found to be effectively inert in the case of pristine graphene. Band structures, Dirac cone, and Fermi level of pristine graphene were found to be unperturbed in the presence of these gases (Figure 2).

On the other hand, a carbon vacancy opens a band gap in graphene and adds two degenerate energy (trap) states, which are $\sim 0.19 \mathrm{eV}$ above the Fermi level (Figure 3a). The valance band maxima (VBM) are also separated from the valance band due to the carbon vacancy. When argon approaches vac. graphene, the degenerate mid gap (trap) states split into two separate energy states (Figure $3 \mathrm{~b}$ ). One state stays near the Fermi energy, while the other moves adjacent to conduction band minima (CBM) and becomes part of the conduction band. Fermi level is moved $\sim 0.01 \mathrm{eV}$ away from CBM due to interaction, which implies drift of electrons from graphene toward argon atoms when a carbon vacancy was present. In the presence of nitrogen, one of the degenerate mid gap (trap) states of vac. graphene disappeared from the forbidden gap, while the other stayed near the Fermi energy level. The energy state separated from the valance band due to the carbon vacancy moves near VBM and becomes part of the valance 

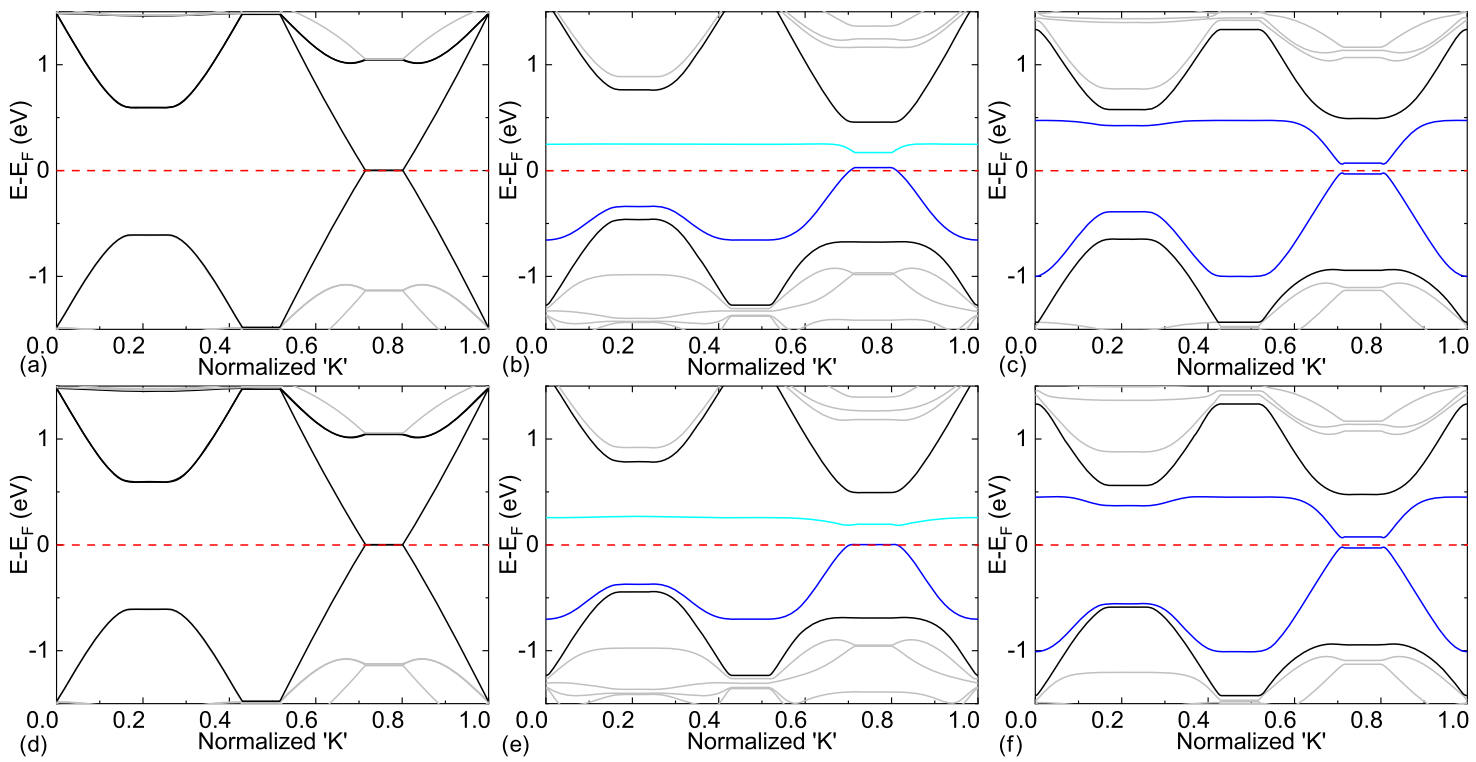

Figure 6. Band structures of a $7 \times 7$ supercell of (a) a pristine graphene $-\mathrm{CO}_{2}$ system, (b) a vac. graphene $-\mathrm{CO}_{2}$ system, (c) an $\mathrm{SW}$ graphene $-\mathrm{CO}$, system, (d) a pristine graphene- $\mathrm{H}_{2} \mathrm{O}$ system, (e) a vac. graphene- $-\mathrm{H}_{2} \mathrm{O}$ system, and (f) an $\mathrm{SW}$ graphene $-\mathrm{H}_{2} \mathrm{O}$ system.
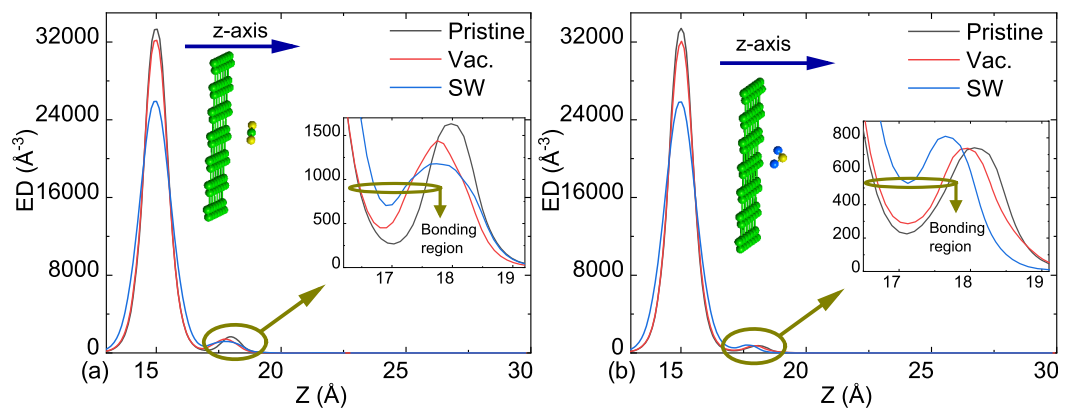

Figure 7. Electron density (ED) comparison of (a) graphene- $\mathrm{CO}_{2}$ and (b) graphene- $\mathrm{H}_{2} \mathrm{O}$ systems for pristine and defective graphene cases. Insets show the respective zoomed-in plots near the overlap regions. The $z$-axis is along the plane normal to graphene's plane and directed toward $\mathrm{CO}_{2}$ and $\mathrm{H}_{2} \mathrm{O}$ atoms.

band. The Fermi energy level moves $\sim 0.12 \mathrm{eV}$ away from CBM adjacent to VBM, which makes the system p-type. Shifting of the Fermi level toward the valance band means an electron drifts from vac. graphene toward nitrogen molecules during the interaction.

A Stone-Wales defect in graphene perturbs the energy states near the Dirac cone and opens a band gap of $\sim 0.05 \mathrm{eV}$ (Figure 4a). When $\mathrm{Ar}$ and $\mathrm{N}_{2}$ approach the SW graphene, they perturb the system further and increase the band gaps to $\sim 0.06$ and $\sim 0.08 \mathrm{eV}$, respectively (Figure $4 \mathrm{~b}, \mathrm{c}$ ). They also separate energy states of CBM and VBM from their conduction and valance bands, respectively.

Electron density (ED), normal to the graphene plane (out of plane, i.e., $z$-axis), has been computed for all of the cases, to probe the consequences and impact of vacancy and SW defects further (Figure 5). ED within the bonding region (i.e., between the graphene plane and the Ar atom) was found to be least influenced by a carbon vacancy, which, however, was enhanced in the presence of an SW defect (Figure 5a, inset). On the other hand, both carbon vacancies and SW defects cause a noticeable increase in ED within the bonding region of the graphene $-\mathrm{N}_{2}$ system (Figure $5 \mathrm{~b}$, inset). An increase in ED within the bonding region signifies an enhanced atomic orbital interaction/overlap between graphene's $\mathrm{C}$ atom and an ambient $\operatorname{Ar}(\mathrm{N} 2)$ atom in the presence of an SW defect (SW or $\mathrm{C}$ vacancy) in graphene.

Using the analysis presented so far, which is based on perturbations in the band structure, Fermi energy, and ED, it can be concluded that while $\mathrm{Ar}$ and $\mathrm{N}_{2}$ are inert to pristine graphene, their inertness toward graphene is compromised in the presence of a carbon vacancy and SW defects. These defects assist in enhanced atomic orbital overlap or orbital interaction, which thereby perturbs the corresponding band structure and Fermi energy, as well as introduces mid gap (trap) states, which collectively can influence the electrical and optoelectronic properties of graphene.

Graphene under $\mathrm{CO}_{2}$ and $\mathrm{H}_{2} \mathrm{O} . \mathrm{CO}_{2}$ and $\mathrm{H}_{2} \mathrm{O}$ are also found to be inert to pristine graphene, while they are found to perturb the band structure as well as Fermi energy of graphene while introducing mid gap (trap) states in the presence of a carbon vacancy and SW defects (Figure 6).

In the presence of $\mathrm{CO}_{2}$ and $\mathrm{H}_{2} \mathrm{O}$, the energy gaps introduced between valance and conduction bands of vac. graphene are modulated to $\sim 0.44$ and $\sim 0.49 \mathrm{eV}$, respectively. They also assist in annihilating one of the degenerate mid gap states of vac. graphene with a shift in Fermi level toward the valance band, which makes these systems p-type (Figure 6b,e). On the other hand, in the presence of $\mathrm{CO}_{2}$ and $\mathrm{H}_{2} \mathrm{O}$, the band gap of SW graphene is increased to $\sim 0.08 \mathrm{eV}$ with a separation 

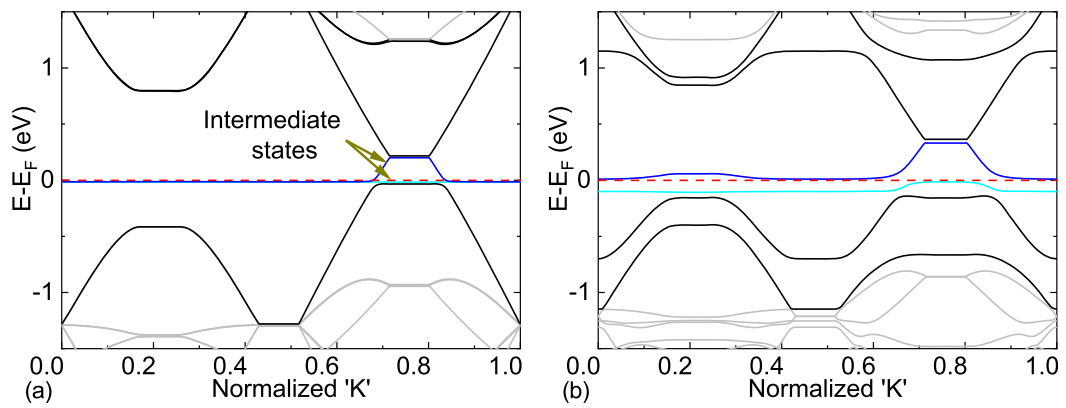

Figure 8. Band structures extracted for a $7 \times 7$ supercell of (a) a pristine graphene $-\mathrm{O}_{2}$ system and (b) an $\mathrm{SW}$ graphene $-\mathrm{O}_{2}$ system.

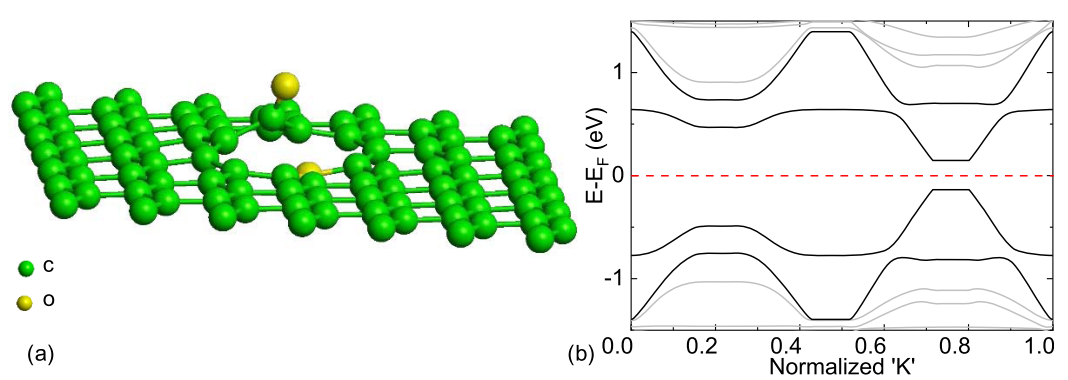

Figure 9. (a) Energy-optimized structure of a $7 \times 7$ supercell of a vac. graphene $-\mathrm{O}_{2}$ system. (b) Band structure of a $7 \times 7$ supercell of a vac. graphene- $\mathrm{O}_{2}$ system.

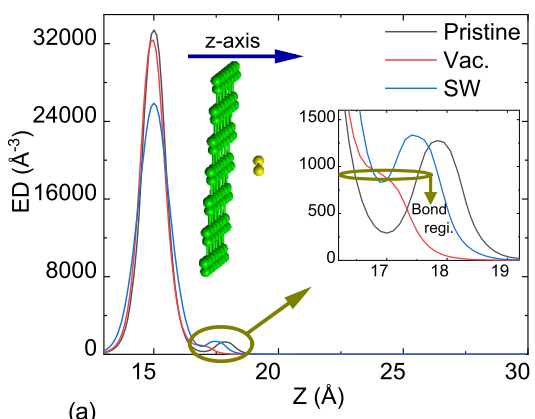

(a)

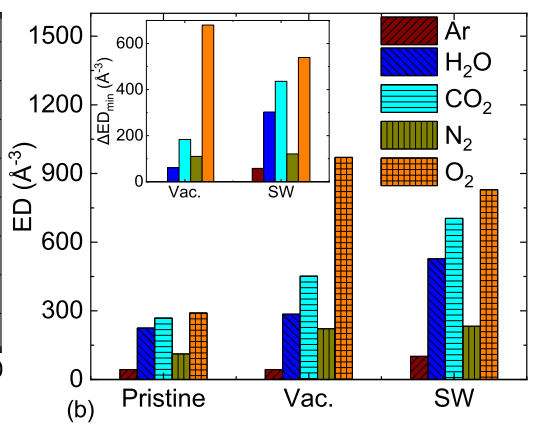

Figure 10. (a) Electron density (ED) comparison of graphene- $\mathrm{O}_{2}$ near the overlap regime. Insets show zoomed-in plots near the overlap regions. The $z$-axis is along the plane normal to graphene's surface, which is directed toward $\mathrm{O}_{2}$. (b) Minimum electron densities (ED) in the bonding regime of different gas-graphene systems. The inset shows the change in the minimum ED of graphene with different gases due to a carbon vacancy and SW defects.

in VBM and CBM from the corresponding valance band and conduction band, respectively ( $6 \mathrm{c}$ and $6 \mathrm{f}$ ), as observed for $\mathrm{N}_{2}$. Moreover, graphene was found to show $\mathrm{p}$-type behavior in the presence of a carbon vacancy and these gases.

$\mathrm{ED}$ in the interface region was found to increase due to a carbon vacancy or SW defects in graphene when $\mathrm{CO}_{2}$ or $\mathrm{H}_{2} \mathrm{O}$ was present around it (Figure 7). The increase in ED signifies that these defects enhance atomic orbital interactions or orbital overlap, thereby improving the chemical bonding of graphene with $\mathrm{CO}_{2}$ and $\mathrm{H}_{2} \mathrm{O}$ in the presence of defects. In comparison to a carbon vacancy defect, an SW defect favors a rather increased atomic orbital overlap (Figure 7, insets), which signifies SW defects to be more sensitive toward these gases.

Why Does Oxygen Cause Degradation? Unlike Ar, $\mathrm{CO}_{2}, \mathrm{H}_{2} \mathrm{O}$, and $\mathrm{N}_{2}, \mathrm{O}_{2}$ perturbs the band structure of pristine graphene. While it preserves the Dirac cone, it introduces new energy states near the Fermi level and moves the Fermi level $\sim 0.21 \mathrm{eV}$ inside the valance band (Figure $8 \mathrm{a}$ ). This makes the system highly p-type. The observed p-type behavior of graphene under ambient oxygen agrees well with previously reported experimental observations. ${ }^{7}$ The introduced energy states near the Dirac cone and Fermi level are further disturbed by an SW defect (Figure 8b). An SW defect further enhances the p-type behavior of graphene in the presence of oxygen, as is evident from the downward shift in Fermi level by $\sim 0.36 \mathrm{eV}$ inside the valance band.

Furthermore, a carbon vacancy was found to play a crucial role in the degradation of graphene under an oxygen environment. Oxygen was found to dissociate near the vacancy site and form a bond with the unsaturation carbon atoms (Figure 9a). One of the dissociated atoms places itself in the graphene plane, whereas the other is moved out of the plane, above the graphene surface. $\mathrm{C}-\mathrm{O}$ bond lengths for these two atoms are 1.38 and $1.23 \AA$, respectively, which are within the $\mathrm{C}-\mathrm{O}$ covalent bond regime. In this process, it releases an energy of $\sim 14.54 \mathrm{eV}$, which apparently is greater than the energy released from the combustion of a single carbon atom $(\sim 10.44 \mathrm{eV})$.

Release of such high energy $(\sim 14.54 \mathrm{eV})$, due to covalent interactions, suggests dissociation of oxygen molecules over the 
vacant site of graphene to be thermodynamically favorable. As a result, the dissociated oxygen turns graphene into graphene oxide locally, rehybridizes the system, and eventually (postgraphene oxide formation) introduces a band gap of $\sim 0.28 \mathrm{eV}$. Keeping these observations in mind, one can suggest that a carbon vacancy seeds dissociation of the graphene layer in the presence of oxygen.

ED along the $z$-axis (Figure 10a) further validates and signifies that a carbon vacancy or an SW defect enhances orbital overlaps between graphene and oxygen. ED of the vac. graphene-oxygen system does not have a minimum present in the bonding regime, which clearly shows the presence of a covalent bond between carbon and oxygen.

Finally, the minimum electron densities inside the bonding region of all studied gases have been compared to understand the relative orbital interaction (Figure 10a). In all of the cases, $\mathrm{O}_{2}$ has the highest affinity (atomic orbital overlap) toward graphene or a defect site. This is followed by $\mathrm{CO}_{2}, \mathrm{H}_{2} \mathrm{O}, \mathrm{N}_{2}$, and $\mathrm{Ar}$ successively. Further, the figure (inset) compares changes in the minimum $\mathrm{ED}$ in the presence of a carbon vacancy and SW defects. A carbon vacancy boosts orbital overlap of graphene with $\mathrm{O}_{2}$, followed by $\mathrm{CO}_{2}, \mathrm{~N}_{2}, \mathrm{H}_{2} \mathrm{O}$, and $\operatorname{Ar}\left(\sim 0.3 \AA^{-3}\right)$ successively. An SW defect also boosts the orbital overlap of all of the gases, which is maximum for $\mathrm{O}_{2}$, followed by $\mathrm{CO}_{2}, \mathrm{H}_{2} \mathrm{O}, \mathrm{N}_{2}$, and $\mathrm{Ar}$ successively.

\section{CONCLUSIONS}

We have investigated interactions of $\mathrm{N}_{2}, \mathrm{O}_{2}, \mathrm{Ar}, \mathrm{CO}_{2}$, and $\mathrm{H}_{2} \mathrm{O}$ with pristine as well as defective (carbon vacancy and SW) graphene. We have found that a carbon vacancy and an SW defect play a significant role in the observed p-type behavior, change in electrical or optoelectronic characteristics, and degradation of graphene under these ambient gases or atmospheric oxygen. Although pristine graphene is chemically inert to $\mathrm{Ar}, \mathrm{CO}_{2}, \mathrm{H}_{2} \mathrm{O}$, and $\mathrm{N}_{2}$, its atomic interaction with graphene is enhanced by the presence of a carbon vacancy or an SW defect. $\mathrm{N}_{2}$ and Ar, which are extensively used for inert environments, are apparently not inert to graphene. Furthermore, their atomic orbital overlaps with graphene are enhanced by these defects, which perturbs the band structure and Fermi energy and introduces mid gap (trap) states in the graphene band structure. $\mathrm{N}_{2}, \mathrm{H}_{2} \mathrm{O}$, and $\mathrm{CO}_{2}$ were found to convert graphene into a p-type semiconductor when a carbon vacancy was present. Pristine graphene was found to become p-type in the presence of $\mathrm{O}_{2}$ and SW defects. Oxygen was also found to be reactive to graphene in the presence of a carbon vacancy. It reacts with unsaturated carbon atoms near the vacant site and degrades graphene by first forming graphene oxide at the defect site and then releasing energy higher than the combustion energy of a single carbon atom. This explains the slow physical degradation of the graphene monolayer in the presence of a vacancy and ambient oxygen.

\section{COMPUTATIONAL DETAILS}

The $\mathrm{DFT}^{36}$-based quantum computational method, supported by QuantumATK package, ${ }^{37,38}$ was used throughout this work.

Computed Modules. For computations, a $7 \times 7$ supercell of (i) pristine graphene, (ii) graphene with a single carbon vacancy (vac. graphene), and (iii) graphene with a single Stone-Wales defect (SW graphene) was considered. These structures with the gas molecules (SI, Figure S2) under study were optimized for minimum energy before creating computation modules to study the interaction among them. To achieve this, respective gas molecules were brought close to the graphene surface/defect before optimizing the structure to achieve the minimum energy conditions. These energyoptimized structures were used to study chemical and physical properties of the systems.

The optimized $\mathrm{C}-\mathrm{C}$ bond length is $\sim 1.43 \AA$, which is very close to the previously reported $\mathrm{C}-\mathrm{C}$ bond length $(1.42 \AA)$ in graphene. Bond lengths of $\mathrm{N}-\mathrm{N}, \mathrm{O}-\mathrm{O}$, and $\mathrm{C}-\mathrm{O}$ in the corresponding molecules are $\sim 1.12, \sim 1.25$, and $\sim 1.18 \AA$, respectively. The $\mathrm{O}-\mathrm{H}$ bond length and the $\mathrm{H}-\mathrm{O}-\mathrm{H}$ bond angle in $\mathrm{H}_{2} \mathrm{O}$ molecules are $\sim 0.98 \AA$ and $\sim 104.45^{\circ}$, respectively. The computed bond lengths and angles are very close to the previously reported values, which validates our computational approach and parameters used.

Computational Parameters. Energy optimizations of all of the simulating modules were done with a $0.01 \mathrm{eV} / \AA$ force and $0.001 \mathrm{eV} / \AA^{3}$ energy cutoffs. The Perdew-BurkeErnzerhof (PBE) form of the generalized gradient approximation (GGA) ${ }^{39}$ functional was used in DFT computations with $5 \times 5 \times 1 k$-point sampling (SI, Figure S1). Periodic boundary conditions were applied in all of the calculations to emulate a large area graphene monolayer. A vacuum region of $\sim 30 \AA$ was added in the supercells perpendicular to the graphene plane to nullify interlayer interactions in the periodic boundary condition. Grimme-D2 van der Waals (vdW) correction $^{40}$ was considered in the calculations to capture long-range vdW interactions. Although more modern dispersion-corrected DFT options, like Tkatchenko-Scheffler ${ }^{41}$ and advance $\mathrm{M} 06 \mathrm{~L},{ }^{42}$ are available, which are very effective especially for large/bulky molecules, ${ }^{43,44}$ Grimme-D2 corrections were used in this work due to computational limitations and relatively smaller sizes of studied molecules. All of the band structures are plotted with normalized " $\kappa$ ", which is along $\mathrm{G} \rightarrow \mathrm{M} \rightarrow \mathrm{L} \rightarrow \mathrm{A} \rightarrow \mathrm{G} \rightarrow \mathrm{K} \rightarrow \mathrm{H} \rightarrow$ A. Positions of $\mathrm{G}, \mathrm{M}, \mathrm{L}$, $\mathrm{A}, \mathrm{G}, \mathrm{K}, \mathrm{H}$, and $\mathrm{A}$ points on the $k$-axis are $0,0.172,0.258$, $0.430,0.516,0.715,0.801$, and 1.0 , respectively. Fermi energy has been set to zero energy level in all of the band structure plots.

\section{ASSOCIATED CONTENT}

\section{Supporting Information}

The Supporting Information is available free of charge at https://pubs.acs.org/doi/10.1021/acsomega.0c04729.

Plot of the $k$-point convergent test; simulating modules of graphene and ambient gas molecules; and minimum energy-optimized simulating molecules (PDF)

\section{AUTHOR INFORMATION}

\section{Corresponding Author}

Mayank Shrivastava - Department of Electronic Systems Engineering, Indian Institute of Science, Bangalore 560012, India; Phone: 09591140309; Email: mayank@iisc.ac.in

\section{Authors \\ Jeevesh Kumar - Department of Electronic Systems Engineering, Indian Institute of Science, Bangalore 560012, India; 1 orcid.org/0000-0001-6178-8434 \\ Ansh - Department of Electronic Systems Engineering, Indian Institute of Science, Bangalore 560012, India}

Complete contact information is available at: https://pubs.acs.org/10.1021/acsomega.0c04729 


\section{Notes}

The authors declare no competing financial interest.

\section{ACKNOWLEDGMENTS}

The authors would like to thank NNetRA program of MeitY, DST, and CSIR, as well as DRDO and MHRD, Government of India, for supporting this work.

\section{REFERENCES}

(1) Novoselov, K. S.; Geim, A. K.; Morozov, S. V.; Jiang, D.; Zhang, Y.; Dubonos, S. V.; Grigorieva, I. V.; Firsov, A. A. Electric Field Effect in Atomically Thin Carbon Films. Science 2004, 306, 666-669.

(2) Morozov, S. V.; Novoselov, K. S.; Katsnelson, M. I.; Schedin, F.; Elias, D. C.; Jaszczak, J. A.; Geim, A. K. Giant Intrinsic Carrier Mobilities in Graphene and Its Bilayer. Phys. Rev. Lett. 2008, 100, 1114.

(3) Balandin, A. A.; Ghosh, S.; Bao, W.; Calizo, I.; Teweldebrhan, D.; Miao, F.; Lau, C. N. Superior Thermal Conductivity of SingleLayer Graphene. Nano Lett. 2008, 8, 902-907.

(4) Lee, C.; Wei, X.; Kysar, J. W.; Hone, J. Measurement of the Elastic Properties and Intrinsic Strength Of Monolayer Graphene. Science 2008, 321, 385-388.

(5) Falkovsky, L. A. Optical Properties of Graphene. J. Phys. Conf. Ser. 2008, 129, No. 012004.

(6) Ramakrishna Matte, H. S. S.; Subrahmanyam, K. S.; Rao, C. N. R. Novel Magnetic Properties of Graphene: Presence of Both Ferromagnetic and Antiferromagnetic Features and Other Aspects. J. Phys. Chem. C 2009, 113, 9982-9985.

(7) Ryu, S.; Liu, L.; Berciaud, S.; Yu, Y. J.; Liu, H.; Kim, P.; Flynn, G. W.; Brus, L. E. Atmospheric Oxygen Binding and Hole Doping in Deformed Graphene on a SiO2 Substrate. Nano Lett. 2010, 10, 49444951.

(8) Liu, L.; Ryu, S.; Tomasik, M. R.; Stolyarova, E.; Jung, N.; Hybertsen, M. S.; Steigerwald, M. L.; Brus, L. E.; Flynn, G. W. Graphene Oxidation: Thickness-Dependent Etching and Strong Chemical Doping. Nano Lett. 2008, 8, 1965-1970.

(9) Shim, J.; Lui, C. H.; Ko, T. Y.; Yu, Y. J.; Kim, P.; Heinz, T. F.; Ryu, S. Water-Gated Charge Doping of Graphene Induced by Mica Substrates. Nano Lett. 2012, 12, 648-654.

(10) Yang, Y.; Brenner, K.; Murali, R. The Influence of Atmosphere on Electrical Transport in Graphene. Carbon 2012, 50, 1727-1733.

(11) Schedin, F.; Geim, A. K.; Morozov, S. V.; Hill, E. W.; Blake, P.; Katsnelson, M. I.; Novoselov, K. S. Detection of Individual Gas Molecules Adsorbed on Graphene. Nat. Mater. 2007, 6, 652-655.

(12) Yuan, W.; Shi, G. Graphene-Based Gas Sensors. J. Mater. Chem. A 2013, 1, 10078-10091.

(13) He, G.; Wang, Q.; Yu, H. K.; Farías, D.; Liu, Y.; Politano, A. Water-Induced Hydrogenation of Graphene/Metal Interfaces at Room Temperature: Insights on Water Intercalation and Identification of Sites for Water Splitting. Nano Res. 2019, 12, 3101-3108.

(14) Politano, A.; Cattelan, M.; Boukhvalov, D. W.; Campi, D.; Cupolillo, A.; Agnoli, S.; Apostol, N. G.; Lacovig, P.; Lizzit, S.; Farías, D.; Chiarello, G.; Granozzi, G.; Larciprete, R. Unveiling the Mechanisms Leading to H2 Production Promoted by Water Decomposition on Epitaxial Graphene at Room Temperature. ACS Nano 2016, 10, 4543-4549.

(15) Larciprete, R.; Colonna, S.; Ronci, F.; Flammini, R.; Lacovig, P.; Apostol, N.; Politano, A.; Feulner, P.; Menzel, D.; Lizzit, S. SelfAssembly of Graphene Nanoblisters Sealed to a Bare Metal Surface. Nano Lett. 2016, 16, 1808-1817.

(16) Chiarello, G.; Fabio, V.; Boukhvalov, D. W.; Politano, A. Sequestration of Carbon Monoxide at Room Temperature at Vacancy Sites of Graphene. Chem. Commun. 2019, 55, 8607-8610.

(17) Grosjean, B.; Pean, C.; Siria, A.; Bocquet, L.; Vuilleumier, R.; Bocquet, M. L. Chemisorption of Hydroxide on 2D Materials from DFT Calculations: Graphene versus Hexagonal Boron Nitride. J. Phys. Chem. Lett. 2016, 7, 4695-4700.
(18) Freitas, R. R. Q.; Rivelino, R.; Mota, F. D. B.; De Castilho, C. M. C. DFT Studies of the Interactions of a Graphene Layer with Small Water Aggregates. J. Phys. Chem. A 2011, 115, 12348-12356.

(19) Al-Hamdani, Y. S.; Alfê, D.; Von Lilienfeld, O. A.; Michaelides, A. Tuning Dissociation Using Isoelectronically Doped Graphene and Hexagonal Boron Nitride: Water and Other Small Molecules. J. Chem. Phys. 2016, 144, No. 154706.

(20) Lee, K. J.; Kim, S. J. Theoretical Investigation of $\mathrm{CO} 2$ Adsorption on Graphene. Bull. Korean Chem. Soc. 2013, 34, 30223026.

(21) Yang, G.; Li, L.; Lee, W. B.; Ng, M. C. Structure of Graphene and Its Disorders: A Review. Sci. Technol. Adv. Mater. 2018, 19, 613648.

(22) Tian, W.; Li, W.; Yu, W.; Liu, X. A Review on Lattice Defects in Graphene: Types Generation Effects and Regulation. Micromachines 2017, 8, No. 163.

(23) Banhart, F.; Kotakoski, J.; Krasheninnikov, A. V. Structural Defects in Graphene. ACS Nano 2011, 5, 26-41.

(24) Kranthi, N. K.; Mishra, A.; Meersha, A.; Variar, H. B.; Shrivastava, M. In Defect-Assisted Safe Operating Area Limits and High Current Failure in Graphene FETs, IEEE International Reliability Physics Symposium (IRPS), March, 2018; pp 3E.11-3E.15.

(25) Mishra, A.; Meersha, A.; Kranthi, N. K.; Trivedi, K.; Variar, H. B.; Veenadhari Bellamkonda, N. S.; Raghavan, S.; Shrivastava, M. In First Demonstration and Physical Insights into Time-Dependent Breakdown of Graphene Channel and Interconnects, IEEE International Reliability Physics Symposium (IRPS), March, 2019; pp 1-6.

(26) Zandiatashbar, A.; Lee, G. H.; An, S. J.; Lee, S.; Mathew, N.; Terrones, M.; Hayashi, T.; Picu, C. R.; Hone, J.; Koratkar, N. Effect of Defects on the Intrinsic Strength and Stiffness of Graphene. Nat. Commun. 2014, 5, No. 3186.

(27) Li, M.; Zhou, H.; Zhang, Y.; Liao, Y.; Zhou, H. The Effect of Defects on the Interfacial Mechanical Properties of Graphene/Epoxy Composites. RSC Adv. 2017, 7, 46101-46108.

(28) Li, M.; Deng, T.; Zheng, B.; Zhang, Y.; Liao, Y.; Zhou, H. Effect of Defects on the Mechanical and Thermal Properties of Graphene. Nanomaterials 2019, 9, No. 347.

(29) Boukhvalov, D. W.; Katsnelson, M. I. Chemical Functionalization of Graphene with Defects. Nano Lett. 2008, 8, 4373-4379.

(30) Li, T.; Yarmoff, J. A. Defect-Induced Oxygen Adsorption on Graphene Films. Surf. Sci. 2018, 675, 70-77.

(31) Ricciardella, F.; Vollebregt, S.; Polichetti, T.; Miscuglio, M.; Alfano, B.; Miglietta, M. L.; Massera, E.; Di Francia, G.; Sarro, P. M. Effects of Graphene Defects on Gas Sensing Properties towards NO2 Detection. Nanoscale 2017, 9, 6085-6093.

(32) Dai, J.; Yuan, J.; Giannozzi, P. Gas Adsorption on Graphene Doped with B, N, Al, and S: A Theoretical Study. Appl. Phys. Lett. 2009, 95, No. 232105.

(33) Ma, J.; Zhang, M.; Dong, L.; Sun, Y.; Su, Y.; Xue, Z.; Di, Z. Gas Sensor Based on Defective Graphene/Pristine Graphene Hybrid towards High Sensitivity Detection of NO2. AIP Adv. 2019, 9, No. 075207.

(34) Zhang, Y. H.; Chen, Y. Bin.; Zhou, K. G.; Liu, C. H.; Zeng, J.; Zhang, H. L.; Peng, Y. Improving Gas Sensing Properties of Graphene by Introducing Dopants and Defects: A First-Principles Study. Nanotechnology 2009, 20, No. 185504.

(35) Ghosh, D.; Pati, S. K. Trapping of Gaseous Pollutants on Defective N-Doped Graphene. Phys. Chem. Chem. Phys. 2017, 19, 636-643.

(36) Kohn, W.; Sham, L. J. Self-consistent equations including exchange and correlation effects. Phys. Rev. 1965, 140, A1133.

(37) Soler, J. M.; Artacho, E.; Gale, J. D.; García, A.; Junquera, J.; Ordejón, P.; Sánchez-Portal, D. The SIESTA Method for Ab Initio Order-N Materials Simulation. J. Phys.: Condens. Matter 2002, 14, $2745-2779$

(38) Synopsys, QuantumATK, Version 2017.2.

(39) Perdew, J. P.; Burke, K.; Ernzerhof, M. Generalized Gradient Approximation Made Simple. Phys. Rev. Lett. 1996, 77, 3865-3868. 
(40) Grimme, S. Semiempirical GGA-type density functional constructed with a long-range dispersion correction. J. Comput. Chem. 2006, 27, 1787-1799.

(41) Bučko, T.; Lebègue, S.; Hafner, J.; Ángyán, J. G. TkatchenkoScheffler van Der Waals Correction Method with and without SelfConsistent Screening Applied to Solids. Phys. Rev. B: Condens. Matter Mater. Phys. 2013, 87, 1-15.

(42) Wang, Y.; Jin, X.; Yu, H. S.; Truhlar, D. G.; He, X. Revised M06-L Functional for Improved Accuracy on Chemical Reaction Barrier Heights, Noncovalent Interactions, and Solid-State Physics. Proc. Natl. Acad. Sci. U.S.A. 2017, 114, 8487-8492.

(43) Johnson, P. S.; Huang, C.; Kim, M.; Safron, N. S.; Arnold, M. S.; Wong, B. M.; Gopalan, P.; Himpsel, F. J. Orientation of a Monolayer of Dipolar Molecules on Graphene from X-Ray Absorption Spectroscopy. Langmuir 2014, 30, 2559-2565.

(44) Joo, Y.; Kim, M.; Kanimozhi, C.; Huang, P.; Wong, B. M.; Singha Roy, S.; Arnold, M. S.; Gopalan, P. Effect of Dipolar Molecule Structure on the Mechanism of Graphene-Enhanced Raman Scattering. J. Phys. Chem. C 2016, 120, 13815-13824. 